\title{
Asymptotic stability of positive fractional 2D linear systems
}

\author{
T. KACZOREK ${ }^{*}$
}

Faculty of Electrical Engineering, Białystok Technical University, 45D Wiejska St., 15-351 Białystok

\begin{abstract}
New necessary and sufficient conditions for the asymptotic stability of the positive fractional 2D systems are established. It is shown that the checking of the asymptotic stability of positive fractional $2 \mathrm{D}$ linear systems can be reduced to testing the stability of corresponding 1D positive linear systems.
\end{abstract}

Key words: asymptotic stability, fractional, positive, 2D linear system.

\section{Introduction}

In positive systems inputs, state variables and outputs take only non-negative values. Examples of positive systems are industrial processes involving chemical reactors, heat exchangers and distillation columns, storage systems, compartmental systems, water and atmospheric pollution models. A variety of models having positive linear behavior can be found in engineering, management science, economics, social sciences, biology and medicine, etc.

Positive linear systems are defined on cones and not on linear spaces. Therefore, the theory of positive systems is more complicated and less advanced. An overview of state of the art in positive systems theory is given in the monographs $[1,2]$.

The most popular models of two-dimensional (2D) linear systems are the models introduced by Roesser [3], FornasiniMarchesini [4, 5] and Kurek [6]. These models have been extended for positive systems in [2, 7, 8]. An overview of positive 2D systems theory has been given in the monograph [2].

Stability of positive $1 \mathrm{D}$ and 2D linear systems has been considered in $[1,8-10]$ and the robust stability in $[11,12]$. The positive fractional linear systems have been addressed in [13-16] and their stability has been investigated in [12, 17-19]. LMI approaches to checking the stability of positive 2D systems have been proposed in [10, 20].

In this paper new necessary and sufficient conditions for the asymptotic stability of the positive fractional 2D linear systems will be established. It will be shown that the checking of the asymptotic stability of positive fractional 2D linear systems can be reduced to testing the stability of corresponding $1 \mathrm{D}$ positive linear systems.

\section{Positive fractional 2D linear systems}

Let $\Re^{n \times m}$ be the set of $n \times m$ real matrices. The set $n \times m$ matrices with nonnegative entries will be denoted by $\Re_{+}^{n \times m}$ and $\Re_{+}^{n}=\Re_{+}^{n \times 1}$. The set of nonnegative integers will be denoted by $Z_{+}$and the $n \times n$ identity matrix will be denoted by $I_{n}$. A matrix $\mathrm{A}=\left[\mathrm{a}_{\mathrm{ij}}\right] \in \Re^{n \times m}$ (a vector $\mathrm{x}$ ) is called strictly positive and denoted by $\mathrm{A}>0(\mathrm{x}>0)$ if and only if $\mathrm{a}_{\mathrm{ij}}>0$ for $\mathrm{i}=1, \ldots, \mathrm{n} ; \mathrm{j}=1, \ldots, \mathrm{m}$.

Definition 1. The $(\alpha, \beta)$ orders fractional difference of an 2D function $x_{i j}$ is defined by the formula

$$
\begin{gathered}
\Delta^{\alpha, \beta} x_{i j}=\sum_{k=0}^{i} \sum_{l=0}^{j} c_{\alpha \beta}(k, l) x_{i-k, j-l}, \\
n_{1}-1<\alpha<n_{1}, \quad n_{2}-1<\beta<n_{2}, \\
n_{1}, n_{2} \in N=\{1,2, \ldots\},
\end{gathered}
$$

where $\Delta^{\alpha, \beta} x_{i j}=\Delta_{i}^{\alpha} \Delta_{j}^{\beta} x_{i j}$ and

$$
\begin{aligned}
& c_{\alpha \beta}(k, l)= \\
& \left\{\begin{array}{c}
1 \text { for } k=0 \text { or } / \text { and } l=0 \\
(-1)^{k+l} \frac{\alpha(\alpha-1) \ldots(\alpha-k+1) \beta(\beta-1) \ldots(\beta-l+1)}{k ! l !} \text { for } k+l>0 .
\end{array}\right.
\end{aligned}
$$

The justification of Definition 1 is given in [21].

Consider the $(\alpha, \beta)$ orders fractional 2D linear system, described by the state equations

$$
\begin{gathered}
\Delta^{\alpha, \beta} x_{i+1, j+1}=A_{0} x_{i j}+A_{1} x_{i+1, j}+A_{2} x_{i, j+1} \\
+B_{0} u_{i j}+B_{1} u_{i+1, j}+B_{2} u_{i, j+1}, \\
y_{i j}=C x_{i j}+D u_{i j},
\end{gathered}
$$

where $x_{i j} \in \Re^{n}, u_{i j} \in \Re^{m}, y_{i j} \in \Re^{p}$ are the state, input and output vectors and $A_{k} \in \Re^{n \times n}, B_{k} \in \Re^{n \times m}, k=0,1,2$, $C \in \Re^{p \times n}, D \in \Re^{p \times m}$.

Using Definition 1 we may write the Eq. (2a) in the form

$$
\begin{gathered}
x_{i+1, j+1}=\bar{A}_{0} x_{i j}+\bar{A}_{1} x_{i+1, j}+\bar{A}_{2} x_{i, j+1} \\
-\sum_{\substack{k=0 \\
k+l>2}}^{i+1} \sum_{\substack{l=0 \\
k+1}} c_{\alpha \beta}(k, l) x_{i-k+1, j-l+1}+B_{0} u_{i j} \\
+B_{1} u_{i+1, j}+B_{2} u_{i, j+1},
\end{gathered}
$$

where $\bar{A}_{0}=A_{0}-I_{n} \alpha \beta, \bar{A}_{1}=A_{1}+I_{n} \beta, \bar{A}_{2}=A_{2}+I_{n} \alpha$.

\footnotetext{
*e-mail: kaczorek@isep.pw.edu.pl
} 
The boundary conditions for the Eq. (3) are given in the form

$$
x_{i 0}, i \in Z_{+} \quad \text { and } \quad x_{0 j}, j \in Z_{+} .
$$

Definition 2. The system (2) is called the (internally) positive fractional 2D system if and only if $x_{i j} \in \Re_{+}^{n}$ and $y_{i j} \in \Re_{+}^{p}$, $i, j \in Z_{+}$for any boundary conditions $x_{0 j} \in \Re_{+}^{n}, j \in Z_{+}$and all input sequences $u_{i j} \in \Re_{+}^{m}, i, j \in Z_{+}, x_{i 0} \in \Re_{+}^{n}, i \in Z_{+}$

It has been shown in [21] that

a) If $0<\alpha<1$ and $1<\beta<2$ then

$$
c_{\alpha \beta}(k, l)<0 \quad \text { for } \quad k=1,2, \ldots ; l=2,3, \ldots
$$

b) If $1<\alpha<2$ and $0<\beta<1$ then

$$
c_{\alpha \beta}(k, l)<0 \quad \text { for } \quad k=2,3, \ldots ; l=1,2, \ldots
$$

Theorem 1. The fractional 2D system (2) for $0<\alpha<1$ and $1<\beta<2$ (or $1<\alpha<2$ and $0<\beta<1$ ) is positive if and only if ${ }^{1}$

$$
\begin{gathered}
\bar{A}_{k} \in \Re_{+}^{n \times n}, \quad B_{k} \in \Re_{+}^{n \times m}, \quad k=0,1,2, \\
C \in \Re_{+}^{p \times n}, \quad D \in \Re_{+}^{p \times m} .
\end{gathered}
$$

The proof is given in [21].

\section{Main results}

Definition 3. The positive fractional 2D linear system (2) is called asymptotically stable if for any bounded boundary conditions $x_{i 0} \in \Re_{+}^{n}, i \in Z_{+} x_{0 j} \in \Re_{+}^{n}, j \in Z_{+}$and zero inputs $\mathrm{u}_{\mathrm{ij}}=0, i, j \in Z_{+}$.

$$
\begin{gathered}
\qquad \lim x_{i j}=0 \\
\text { for all } \quad x_{i 0} \in \Re_{+}^{n}, \quad i, j \rightarrow \infty, \\
i \in Z_{+} x_{0 j} \in \Re_{+}^{n}, \quad j \in Z_{+}
\end{gathered}
$$

In the proof of the main result of this paper the following lemma and theorem will be used.

Lemma. If $0<\alpha<1$ and $1<\beta<2$ (or $1<\alpha<2$ and $0<\beta<1$ ) then

$$
\sum_{k=0}^{\infty} \sum_{l=0}^{\infty} c_{\alpha \beta}(k, l)=0
$$

Proof. It is well-known [17] that

$$
\begin{gathered}
\sum_{i=0}^{\infty}(-1)^{i}\left(\begin{array}{c}
\alpha \\
i
\end{array}\right)=\sum_{i=0}^{\infty}(-1)^{i} \frac{\alpha(\alpha-1) \ldots(\alpha-i+1)}{i !}=0 \\
\text { for } \quad \alpha>0 .
\end{gathered}
$$

\footnotetext{
${ }^{1}$ It is assumed that $\sum_{k=2}^{i+1} c_{\alpha \beta}(k, l) x_{i-k+1, j}=0, \sum_{l=2}^{j+1} c_{\alpha \beta}(0, l) x_{i+1, j-l+1}=0$.
}

Proof is given in $[9,19]$.

Theorem 3. The positive fractional 2D system (2) is asymptotically stable if and only if the positive 1D system

$x_{i+1}=\left(\hat{A}+I_{n}\right) x_{i}, \quad \hat{A}=A_{0}+A_{1}+A_{2}, \quad x_{i} \in R_{+}^{n}, \quad i \in Z_{+}$

is asymptotically stable.

Proof. From (3) for $B_{0}=B_{1}=B_{2}=0$ we have

$$
\begin{gathered}
x_{i+1, j+1}=A_{0} x_{i j}+A_{2} x_{i+1, j}+A_{2} x_{i, j+1} \\
+\sum_{\substack{k=0 \\
k+l>0}}^{i+1} \sum_{\substack{l=0 \\
j+1}} c_{k l} x_{i-k+1, j-l+1}
\end{gathered}
$$

where $\mathrm{c}_{k l=} c_{\alpha \beta}(k, l)$.

By Theorem 7 the positive $2 \mathrm{D}$ system with delays is asymptotically stable if and only if the positive $1 \mathrm{D}$ system

$x_{i+1}=\left(\hat{A}+\sum_{\substack{k=0 \\ k+l>0}}^{\infty} \sum_{\substack{l=0 \\ \infty}}^{\infty} c_{k l} I_{n}\right) x_{i}, \quad x_{i} \in R_{+}^{n}, \quad i \in Z_{+}$

is asymptotically stable. From (1b) we have $c_{00}=$ $-c_{\alpha \beta}(0,0)=-1$ and from (8) we obtain 


$$
\sum_{\substack{k=0 \\ k+l>0}}^{\infty} \sum_{\substack{l=0 \\ k}}^{\infty} c_{k l}=I_{n}
$$

Substitution of (15) into (14) yields (13).

Applying to the positive 1D system the well-known theorem $[9,18]$ we obtain the following theorem.

Theorem 4. The positive fractional $2 \mathrm{D}$ system (2) is asymptotically stable if and only if one of the following equivalent conditions holds:

1. Eigenvalues $z_{1}, \ldots, z_{n}$ of the matrix $\hat{A}+I_{n}$ have moduli less than 1 ,

2. All coefficients of the characteristic polynomial of the matrix $\hat{A}$ are positive,

3. All leading principal minors of the matrix $-\hat{A}$ are positive.

Theorem 5. The positive fractional $2 \mathrm{D}$ system (2) is unstable if at least one diagonal entry of the matrix $\hat{A}$ is positive.

Proof. If at least one diagonal entry of the matrix $\hat{A}$ is positive then at least one diagonal entry of the matrix $\hat{A}+I_{n}$ is greater than 1 and it is well-known [2, 17-18, 22-23] that the system (13) is unstable.

Example 1. Using Theorem 4 check the asymptotic stability of the positive fractional 2D system (2) for $\alpha=0.3$ and $\beta=1.2$ with the matrices

$$
\begin{gathered}
A_{0}=\left[\begin{array}{cc}
0.4 & 0 \\
0.1 & 0.5
\end{array}\right], \\
A_{1}=\left[\begin{array}{cc}
-1 & 0 \\
0.2 & -1.1
\end{array}\right], \\
A_{2}=\left[\begin{array}{cc}
-0.2 & 0 \\
0.1 & -0.3
\end{array}\right] .
\end{gathered}
$$

Note that the fractional system is positive since the matrices

$$
\begin{gathered}
\bar{A}_{0}=A_{0}-I_{n} \alpha \beta=\left[\begin{array}{cc}
0.04 & 0 \\
0.1 & 0.14
\end{array}\right], \\
\bar{A}_{1}=A_{1}+I_{n} \beta=\left[\begin{array}{cc}
0.2 & 0 \\
0.2 & 0.1
\end{array}\right], \\
\bar{A}_{2}=A_{2}+I_{n} \alpha=\left[\begin{array}{ll}
0.1 & 0 \\
0.1 & 0
\end{array}\right]
\end{gathered}
$$

have nonnegative entries.

In this case

$$
\hat{A}=\bar{A}_{0}+\bar{A}_{1}+\bar{A}_{2}=\left[\begin{array}{cc}
-0.8 & 0 \\
0.4 & -0.9
\end{array}\right] .
$$

The first condition of Theorem 4 is satisfied since the matrix

$$
\hat{A}+I_{n}=\left[\begin{array}{cc}
0.2 & 0 \\
-0.4 & 0.1
\end{array}\right]
$$

has the eigenvalues $z_{1}=0.2, z_{2}=0.1$ whose moduli are less than 1 .
The second condition of Theorem 4 is also satisfied since characteristic polynomial of the matrix (21)

$$
\operatorname{det}\left[I_{n} z-\hat{A}\right]=\left|\begin{array}{cc}
z+0.8 & 0 \\
-0.4 & z+0.9
\end{array}\right|=z^{2}+1.7 z+0.72
$$

has positive coefficients.

All leading principles minors of the matrix

$$
-\hat{A}=\left[\begin{array}{cc}
0.8 & 0 \\
-0.4 & 0.9
\end{array}\right]
$$

are positive $\Delta_{1}=0.8, \Delta_{2}=0.72$.

Therefore, all three conditions of Theorem 4 are satisfied and the positive fractional 2D system with the matrices (17) is asymptotically stable.

Example 2. Using Theorem 5 we will shown that the positive fractional 2D system (2) for $\alpha=0.5$ and $\beta=1.2$ with the matrices

$$
\begin{gathered}
A_{0}=\left[\begin{array}{ll}
0.6 & 0.1 \\
0.1 & 0.7
\end{array}\right], \\
A_{1}=\left[\begin{array}{cc}
-0.1 & 0.3 \\
0 & -0.2
\end{array}\right], \\
A_{2}=\left[\begin{array}{cc}
-0.4 & 0.2 \\
0 & -0.5
\end{array}\right]
\end{gathered}
$$

is unstable.

In this case the matrix

$$
\hat{A}=A_{0}+A_{1}+A_{2}=\left[\begin{array}{cc}
0.1 & 0.6 \\
0 & 0
\end{array}\right],
$$

has one positive diagonal entry. Therefore, by Theorem 5 the positive fractional system is unstable. The same result we obtain using one of the conditions of Theorem 4.

\section{Concluding remarks}

New necessary and sufficient conditions for the asymptotic stability of the positive fractional 2D systems have been established. Simple sufficient conditions for instability of the system have been also given. It has been shown that checking the asymptotic stability of the positive fractional 2D linear systems can be reduced to testing the stability of corresponding 1D positive linear systems.

Assuming in (1a) $B_{1}=B_{2}=0$ we obtain the first Fornasini-Marchesisni model and assuming $A_{0}=0$ and $B_{0}=0$ we obtain the second Fornasini-Marchesisni model.

Therefore, the considerations for the first and second Fornasini-Marchesini models are particular case of the presented general 2D model.

The considerations presented for the general model can be easily extended for the Roesser model. Extensions of those considerations for the hybrid positive $2 \mathrm{D}$ linear systems and for $2 \mathrm{D}$ continuous-time linear systems are open problems. 
Acknowledgements. This work was supported by Ministry of Science and Higher Education in Poland under the grant No NN514 193933.

\section{REFERENCES}

[1] L. Farina and S. Rinaldi, Positive Linear Systems; Theory and Applications, J. Wiley, New York, 2000.

[2] T. Kaczorek, Positive $1 D$ and $2 D$ Systems, Springer-Verlag, London, 2002.

[3] R.P. Roesser, "A discrete state-space model for linear image processing", IEEE Trans. Autom. Contr. AC-20 (1), 1-10 (1975).

[4] E. Fornasini and G. Marchesini, "State-space realization theory of two-dimensional filters", IEEE Trans. Autom. Contr. AC-21, 484-491 (1976).

[5] E. Fornasini and G. Marchesini, "Double indexed dynamical systems", Math. Sys. Theory 12, 59-72 (1978).

[6] J. Kurek, "The general state-space model for a two-dimensional linear digital systems", IEEE Trans. Autom. Contr. AC-30, 600-602 (1985).

[7] T. Kaczorek, "Reachability and controllability of non-negative 2D Roesser type models", Bull. Pol. Ac.: Tech. 44 (4), 405-410 (1966).

[8] M.E. Valcher, "On the initial stability and asymptotic behavior of 2D positive systems", IEEE Trans. on Circuits and Systems 44 (7), 602-613 (1977).

[9] T. Kaczorek, "Asymptotic stability of positive 1D and 2D linear systems", Recent Advances in Control and Automation 1, 41-52 (2008).

[10] M. Twardy, "An LMI approach to checking stability of 2D positive systems", Bull. Pol. Ac.: Tech. 55 (4), 385-393 (2007).

[11] M. Busłowicz, "Robust stability of positive discrete-time linear systems with multiple delays with unity rank uncertainty structure or non-negative perturbation matrices", Bull. Pol. Ac.: Tech. 55 (1), 347-350 (2007).
[12] M. Busłowicz, "Robust stability of convex combination of two fractional degree characteristic polynomials", Acta Mechanica et Automatica 2 (2), 5-10 (2008).

[13] T. Kaczorek, "Reachability and controllability to zero tests for standard and positive fractional discrete-time systems", J. Automation and System Engineering, (2009), (to be published).

[14] T. Kaczorek, "Reachability and controllability to zero of positive fractional discrete-time systems", Machine Intelligence and Robotic Control 6 (4), 411-413 (2007).

[15] T. Kaczorek, "Reachability and controllability to zero of cone fractional linear systems", Archives of Control Sciences 17 (3), 357-367 (2007).

[16] T. Kaczorek, "Fractional positive continuous-time linear systems and their reachability", Int. J. Appl. Math. Comput. Sci. 18 (2), 223-228 (2008).

[17] M. Busłowicz and T. Kaczorek, "Simple conditions for practical stability of positive fractional discrete-time linear systems", J. Automation and System Engineering, (2009), (to be published).

[18] T. Kaczorek, "Practical stability of positive fractional 2D linear systems", Bull. Pol. Ac.: Tech. 56 (4), 405-410 (2008).

[19] T. Kaczorek, "Independence of the asymptotic stability of the 2D linear systems with delays of their delays", Int. J. Appl. Math. Comput. Sci. 19, (2009), (to be published).

[20] T. Kaczorek, "LMI approach to stability of 2D positive systems with delays", Multidimensional Systems and Signal Processing 18 (3), 277-279 (2008).

[21] T. Kaczorek, "Positive different orders fractional 2D linear systems", Acta Mechanica et Automatica 2 (2), 51-58 (2008).

[22] N.K. Bose, Multidimensional Systems Theory Progress, Directions and Open Problems, Reidel Publishing Co., Boston, 1985.

[23] T. Kaczorek, "Asymptotic stability of positive 2D linear systems", $13^{\text {th }}$ Proc. Scientific Conf. Computer Applications in Electrical Engineering 4, 14-16 (2008). 